\title{
Gas foaming of electrospun poly(L-lactide-co-caprolactone)/silk fibroin nanofiber scaffolds to promote cellular infiltration and tissue regeneration
}

\author{
Yujie Chen ${ }^{\mathrm{a}, 1}$, Zihao Jia ${ }^{\mathrm{b}, 1}$, Muhammad Shafiq ${ }^{\mathrm{c}}$, Xianrui Xie ${ }^{\mathrm{a}}$, Xianghao Xiao ${ }^{\mathrm{a}}$, Rita Castro ${ }^{\mathrm{d}}$, \\ João Rodrigues ${ }^{\mathrm{d}}$, Jinglei $\mathrm{Wu}^{\mathrm{a}}$, Guangdong Zhou ${ }^{\mathrm{b}, *}$, Xiumei Mo ${ }^{\mathrm{a}, *}$ \\ ${ }^{a}$ College of Chemistry, Chemical Engineering and Biotechnology, Donghua University, China \\ ${ }^{\mathrm{b}}$ Department of Plastic and Reconstructive Surgery, Shanghai Key Lab of Tissue Engineering, Shanghai 9th People's Hospital, Shanghai Jiao Tong University School of \\ Medicine, Shanghai, China \\ ${ }^{\mathrm{c}}$ Department of Chemistry, Pakistan Institute of Engineering \& Applied Sciences (PIEAS), Nilore, 45650, Islamabad, Pakistan \\ d CQM- Centro de Química da Madeira, MMRG, Universidade da Madeira, Campus Universitário da Penteada, 9020-105, Funchal, Portugal
}

\section{A R T I C L E I N F O}

\section{Keywords:}

Electrospun nanofiber scaffold

Gas foaming

Three-dimensional scaffolds

Cell infiltration

Tissue engineering

\begin{abstract}
A B S T R A C T
Electrospun nanofibers emulate extracellular matrix (ECM) morphology and architecture; however, small pore size and tightly-packed fibers impede their translation in tissue engineering. Here we exploited in situ gas foaming to afford three-dimensional (3D) poly(L-lactide-co-e-caprolactone)/silk fibroin (PLCL/SF) scaffolds, which exhibited nanotopographic cues and a multilayered structure. The addition of SF improved the hydrophilicity and biocompatibility of 3D PLCL scaffolds. Three-dimensional scaffolds exhibited larger pore size (38.75 $\left.\pm 9.78 \mu \mathrm{m}^{2}\right)$ and high porosity $(87.1 \% \pm 1.5 \%)$ than that of their $2 \mathrm{D}$ counterparts. 3D scaffolds also improved the deposition of ECM components and neo-vessel regeneration as well as exhibited more numbers of $\mathrm{CD}_{163}{ }^{+} /$ $\mathrm{CCR7}^{+}$cells after 2 weeks implantation in a subcutaneous model. Collectively, 3D PLCL/SF scaffolds have broad implications for regenerative medicine and tissue engineering applications.
\end{abstract}

\section{Introduction}

Electrospinning has gathered significant attention from the research community owing to its potential to afford extracellular matrix (ECM) mimetic nanofibers. Due to high porosity and large surface area-tovolume ratio, electrospun scaffolds find extensive applications in regenerative medicine and tissue engineering (TE). However, scaffolds assembled from electrospinning are generally 2D nanofibrous membranes, such as sheet-like structures and tubular structures, which comprise of densely packed fiber layers with only superficial pores. The small pore size resulting from tightly-packed nanofibers hinder cellular infiltration and diffusion of growth nutrients at the microstructural level and the tissue formation into electrospun scaffolds [1,2]. Thus, it is pertinent to devise an innovative strategy to afford electrospun scaffolds with improved porosity and loosely-packed fibers structure, while exhibiting nanofibrous morphology and an appropriate pore size [3].

To circumvent these limitations and afford 3D scaffolds, an array of approaches, including the use of sacrificial fibers, electrospraying technique, post-treatment of fibers as well as liquid- and template- assisted collection have been put forwarded [4-8]. However, these approaches face several shortcomings, including the use of special instrumentation, lengthy and time-consuming experimental procedures, and poor control over the morphology of the scaffolds, necessitating the simple, controllable, and effective method for generating 3D scaffolds.

The gas foaming methods, which either utilize the in situ generation of gas bubbles via a chemical reaction (e.g., the decomposition of sodium borohydride, $\mathrm{NaBH}_{4}$ or ammonium bicarbonate) or the addition of inert gases $\left(\mathrm{CO}_{2}\right.$ or $\left.\mathrm{H}_{2}\right)$ have received significant attention to afford 3D porous scaffolds [9-11]. Joshi et al. [12] fabricated three-dimensional (3D) nanofibers scaffolds by immersing 2D electrospun membranes into $\mathrm{NaBH}_{4}$ solution. Yet in another seminal study, Gao et al. [13] treated 2D electrospun membranes with $\mathrm{NaBH}_{4} /$ methanol solution, which yielded 3D porous scaffolds. These approaches have successfully produced 3D nanofibrous scaffolds with porous or multilayered structures, suggesting their potential use for cell infiltration and growth into scaffolds.

Despite obvious advantages including the expansion of nanofibers into 3D scaffolds, limitations associated with hydrophobic polymers (polycaprolactone, poly(vinylene difluoride), poly(L-lactide-co-

\footnotetext{
* Corresponding authors.

E-mail addresses: guangdongzhou@126.com (G. Zhou), xmm@dhu.edu.cn (X. Mo).

1 These authors contributed equally to this work.
} 
$\varepsilon$-caprolactone) (PLCL), nylon, etc.) in achieving 3D-like architecture and cell recognizability underspin the translation of the gas foaming technology $[12,14]$. On the other hand, natural polymers exhibit good biocompatibility and cell adhesion motifs; however, they exhibit weak mechanical properties and necessitate crosslinking to realize mechanical stability in aqueous solution during the foaming [15]. Hybrid scaffolds consisting of natural and synthetic polymers may obviate limitations associated with either natural or synthetic polymers. However, to the best of the authors' knowledge, there is a paucity of hybrid 3D scaffolds. The objective of this study is therefore to fabricate gas-foamed 3D-like hybrid scaffolds. PLCL, a mechano-elastic and biodegradable polymer, and silk fibroin (SF), a hydrophilic and biocompatible polymer were used to assemble aligned nanofibrous membranes and expanded thereafter [16]. It is noteworthy that the degradation products of SF are safe with a low inflammatory response. Previously, PLCL/SF scaffolds exhibited good biocompatibility, mechanical properties, hydrophilicity, and were extended for vascular remodeling, nerve regeneration, and tendon healing [17-20]. Here, the aligned 2D nanofibrous membranes composed of PLCL and SF (2DNFS) were fabricated, which were then subjected to gas foaming to afford 3D scaffolds (3DNFS). The hydrophobic nature of PLCL may hinder the filing of the foaming solution into the nano/micropores of the membrane [21]. We hypothesize that the incorporation of SF into the scaffolds will overcome the hydrophobic nature of PLCL and afford scaffolds with improved mechanical properties, biodegradability, and biocompatibility. Moreover, surface morphology, surface roughness, mechanical properties, and porosity were evaluated. It is foreseen that the 3DNFS could promote cellular infiltration and neo-tissue regeneration.

\section{Experimental}

\subsection{Materials}

PLCL $(\mathrm{Mw}=300 \mathrm{kDa})$ with an $\mathrm{L}_{\mathrm{L}}$-lactic acid/ $\varepsilon$-caprolactone ratio of 50:50 was supplied by Jinan Daigang Biomaterial Co., Ltd. (Jinan, China). Cocoon was purchased from Jiaxing Silk Co. (China). 1,1,1,3,3,3-hexafluoro-2-propanol (HFIP) was purchased from Shanghai Darui Fine Chemical Co., Ltd. (Shanghai, China). Glutaraldehyde was obtained from Aladdin Bio-Chem Technology Co., Ltd. (Shanghai, China). $\mathrm{NaBH}_{4}$ was supplied by Sinopharm Chemical Reagent Co., Ltd (Shanghai, China). All chemicals were of reagent grade and used as received without any further purification or modification.

\subsection{Preparation of PLCL membrane and 2DNFS}

PLCL was dissolved in HFIP at a concentration of $10 \%(\mathrm{w} / \mathrm{v})$. PLCL and SF with a ratio of 8:2 $(\mathrm{w} / \mathrm{w})$ were dissolved in HFIP at a concentration of $10 \%(\mathrm{w} / \mathrm{v})$. Then, the polymer blend solutions were transferred into a $10-\mathrm{mL}$ syringe with a $20 \mathrm{G}$ stainless steel needle and pumped at a flow rate of $1.2 \mathrm{~mL} / \mathrm{h}$ with $12 \mathrm{kV}$ of an applied voltage. The 2D PLCL/SF nanofiber scaffolds (2DNFS) were collected on a drum collector with rotating speeds of $2200 \mathrm{rpm}$ at a distance of $12 \mathrm{~cm}$ from the needle. PLCL membrane was prepared by the same method and parameters. Then the PLCL membrane and 2DNFS were vacuum dried in an oven for 3 days to remove any residual solvent. 2DNFS were subjected to a desiccator containing glutaraldehyde vapor for $6 \mathrm{~h}$. The crosslinked scaffolds were soaked in glutamic acid solution to remove residual glutaraldehyde. Next, the wet 2DNFS was put into the refrigerator at $-80{ }^{\circ} \mathrm{C}$ for $24 \mathrm{~h}$ and subsequently transferred to a vacuum freeze dryer for $72 \mathrm{~h}$ to obtain the crosslinked and dried 2DNFS.

\subsection{Preparation of $3 D N F S$}

Dried 2DNFS were immersed into the freshly prepared aqueous solution of $\mathrm{NaBH}_{4}(1 \mathrm{M})$. The hydrogen generation is produced according to Eq. (1):
$\mathrm{NaBH}_{4}+2 \mathrm{H}_{2} \mathrm{O} \rightarrow \mathrm{NaBO}_{2}+4 \mathrm{H}_{2} \uparrow$.

The hydrogen bubbles evolution will converge into the internal pore space of the 2DNFS, a process that will result in the expansion in the thickness and volume of the 2D membranes into a fully developed 3D nanofiber scaffold (3DNFS). After $1 \mathrm{~h}$, the 3DNFS were removed from the solution and washed with deionized water three times to remove the residual $\mathrm{NaBH}_{4}$. In the same way, 3DNFS were kept in a refrigerator at $-80{ }^{\circ} \mathrm{C}$ for freezing and then transferred to a vacuum freeze dryer to afford stable porous structure. The swelling degree of 2DNFS and 3DNFS was measured by incubating the scaffolds in PBS solution (refer to the supplementary information). The mechanical properties were also measured by incubating the scaffolds in PBS for up to $2 \mathrm{~h}$ (please see the supporting informating). The surface roughness of the scaffolds was also measured by atomic force microscopy (please see supplementary information for a detailed procedure).

\subsection{In vitro cytocompatibility assay}

NIH 3T3 fibroblasts were maintained and expanded in high glucose Dulbecco's modified eagle medium (DMEM) with $10 \%$ fetal bovine serum (FBS), 1\% penicillin (100 units/mL), and streptomycin (100 $\mu \mathrm{g} /$ $\mathrm{mL}$ ). The NIH 3T3 fibroblasts were seeded onto different scaffolds (one scaffold in each well of 48 -well plates and at $1 \times 10^{4}$ cells/scaffold, the circular 2DNFS and 3DNFS are both $10 \mathrm{~mm}$ in diameter, about $0.005 \mathrm{~g}$ in mass, $0.1 \mathrm{~mm}$ and $3 \mathrm{~mm}$ in thickness, respectively). The culture medium was removed and replaced with $600 \mu \mathrm{l}$ of fresh medium after every 2 days.

The cell cytoskeleton organization was determined by staining Factin and nuclei with phalloidin and 4',6-diamidino-2-phenylindole (DAPI). NIH-3T3 fibroblasts were cultured on 2DNFS and 3DNFS for $3 \mathrm{~d}$. Then, remove the medium, and cells were washed with PBS three times and fixed with paraformaldehyde (PFA) for $2 \mathrm{~h}$. After fixation, cells were washed with PBS three times and permeabilized with $0.1 \%$ Triton for 10 min, washed three times with PBS, and incubated with $2 \%$ BSA solution for $30 \mathrm{~min}$. Afterward, cells were washed with PBS and stained with phalloidin for $30 \mathrm{~min}$, followed by washing with PBS three times and staining with DAPI for $10 \mathrm{~min}$. The cytoskeleton was then observed under a confocal laser scanning microscope (Nikon, A1RMP, Japan).

The cell cytotoxicity/proliferation of samples was determined by cell counting kit 8 (CCK-8). Briefly, the culture medium was removed after 1 , 3 , and $5 \mathrm{~d}$ of cell culture, then scaffolds were washed with PBS for three times and incubated with the $200 \mu$ Lof the fresh medium containing 20 $\mu \mathrm{L}$ of CCK- 8 and $180 \mu \mathrm{Lof}$ serum-free medium per well. After incubation at $37{ }^{\circ} \mathrm{C}$ for $2 \mathrm{~h}, 100 \mu \mathrm{L}$ of the medium was transferred into a 96-well plate to measure the absorbance at a wavelength of $450 \mathrm{~nm}$.

The cell morphology was characterized by Phenom XL SEM (Phenom Scientific Instruments Co. Ltd., Shanghai, China). After 5 days of cell culture, the medium was removed and scaffolds were washed with PBS three times. For fixation, the scaffold was soaked with PFA for $2 \mathrm{~h}$, washed with PBS three times, and then dehydrated with alcohol gradient (30\%, 50\%, 60\%, 70\%, 80\%, 90\%, and 100\%) for $30 \mathrm{~min}$ each. After drying in the fume hood, the morphology of cells on the scaffold was observed by SEM.

Cell infiltration was evaluated by histological staining. After 5 days of culture, cells were fixed by soaking the scaffold with PFA for $24 \mathrm{~h}$, dehydrated through a series of ethanol solutions as previously described. The scaffold was embedded in paraffin to obtain cross-sections and stained with hematoxylin and eosin (H\&E). Finally, the infiltration of cells within the scaffolds was observed under an optical microscope.

\subsection{In vivo studies}

All animals were from Shanghai Jiao Tong University (SJTU) and treated according to the standard guidelines approved by the ethics committee of SJTU. 2DNFS and 3DNFS with a diameter of $1.5 \mathrm{~cm}$ were 
A
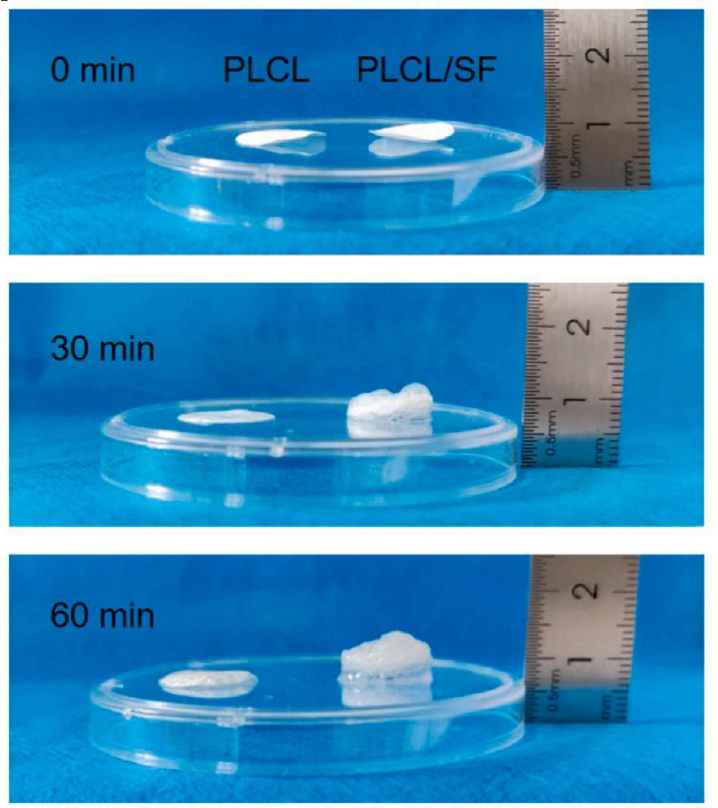

B
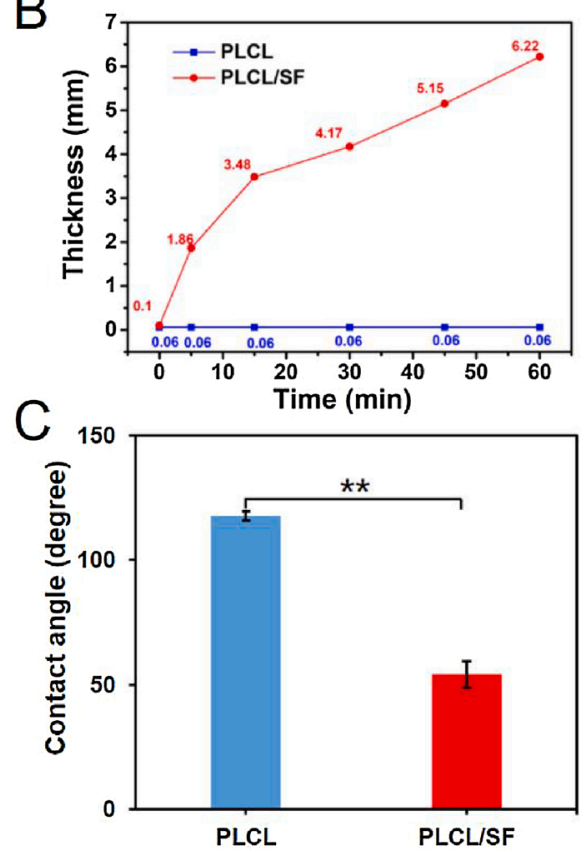

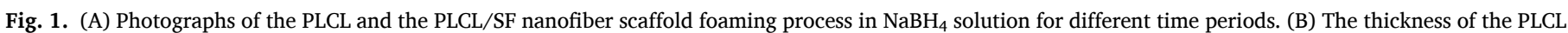

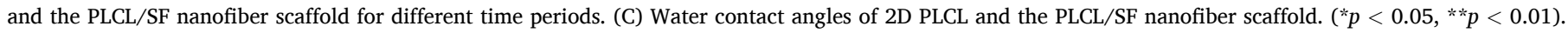

sterilized with ethylene oxide gas before implantation. 9-week-old SD rats (weight, 250-300 g) were anesthetized with $4 \%$ isoflurane for about $2 \mathrm{~min}$. After shaving $4 \times 4 \mathrm{~cm}^{2}$ area on the back of each rat, the exposed skin was disinfected with a povidone-iodine solution. An incision of $2 \mathrm{~cm}$ was made on the back of each rat.One 2DNFS and one 3DNFS with a diameter of $1.5 \mathrm{~cm}$ and thickness of $3 \mathrm{~mm}$ were inserted into the subcutaneous pocket of each rat. The rats were anesthetized and euthanized with $\mathrm{CO}_{2}$ at $1,2,4$ weeks post-implantation. The explants with the surrounding tissues were retrieved from the subcutaneous pocket and fixed in 4\% PFA for up to $3 \mathrm{~d}$. The fixed samples were dehydrated through an alcohol gradient (70-100\%), embedded in paraffin, and cut into $4-\mu \mathrm{m}$ sections $(4 \mu \mathrm{m})$. Subsequently, the sections were stained with $\mathrm{H} \& \mathrm{E}$ and Masson trichrome.

Immunofluorescence staining for DAPI, von Willebrand Factor (VWF), and CD 163 was applied to observe cell infiltration, neovascularization, and macrophage. Briefly, tissue sections were deparaffinized, blocked, and incubated with the primary antibody overnight at $4{ }^{\circ} \mathrm{C}$; they were afterward incubated with the fluorescent-labeled secondary antibody at room temperature for $50 \mathrm{~min}$. The nuclei were stained with DAPI for $5 \mathrm{~min}$ and rinsed three times.

Immunohistochemical staining for CCR7, CD163, CD68 was used to characterize the macrophage phenotypes and inflammatory response on 2DNFS and 3DNFS. The previously prepared sections were placed in a repair box filled with citrate buffer $(\mathrm{pH}=6.0)$ antigen repair solution for $20 \mathrm{~min}$. The slices were then incubated in $3 \%$ hydrogen peroxide $\left(\mathrm{H}_{2} \mathrm{O}_{2}\right)$ solution at room temperature for 25 min to block endogenous peroxidase and prevent non-specific antibody binding with blocking solution ( $2 \%$ normal goat serum, $0.1 \%$ Triton X-100 in PBS). Next, the diluted primary antibody (1:200) was dropped onto the slides and incubated at $4{ }^{\circ} \mathrm{C}$ overnight. The sections were washed and incubated with the horseradish peroxidase (HRP) conjugated secondary antibodies at room temperature for $50 \mathrm{~min}$. The 3, 3'-diaminobenzidine (DAB) was then added and terminated the reaction when the positive staining turned brown.

Microscopic images of H\&E staining, Masson's trichrome staining, immunohistochemical staining, and immunofluorescence staining were all obtained with a microscope (Leica Microsystems, Germany). The depth of cell and collagen infiltration, capsule thickness, and the number of blood vessels and macrophage phenotypes were measured using Image-Pro Plus software (Media Cybernetics, Inc., Bethesda, MD, USA).

\subsection{Statistical analysis}

All the data were obtained at least in triplicate, and the values were expressed as mean \pm standard deviation (SD). To test the significance of observed differences between the study groups, an unpaired Student $t$ test was applied. A value of $\left(^{*}\right) p<0.05$ and $(* *) p<0.01$ were considered to be significant and highly significant, respectively.

\section{Results}

\subsection{Fabrication and characterization of PLCL/SF scaffolds}

As shown in Fig. 1A and B, PLCL and PLCL/SF scaffolds are of similar thickness $(0.06 \mathrm{~mm}$ and $0.1 \mathrm{~mm})$ before foaming. During the foaming process, the thickness of the PLCL scaffold did not increase significantly, while the thickness of the PLCL/SF scaffold reached $4.17 \mathrm{~mm}$ at $30 \mathrm{~min}$ and $6.22 \mathrm{~mm}$ at $60 \mathrm{~min}$. Intriguingly, pure PLCL membranes cannot be fabricated into 3D scaffolds in the $\mathrm{NaBH}_{4}$ solution owing to their hydrophobic nature. SF enhanced the hydrophilicity of the scaffold (Fig. 1C) and its thickness increased significantly after the expansion process. Because $\mathrm{NaBH}_{4}$ solution was easily absorbed into PLCL/SF nanofiber membrane and followed by the generation of $\mathrm{H}_{2}$ gas bubbles in situ, which rearranged the fibers into a 3D-like architecture by exerting pressure on the surrounding fibers, resulting in a multilayered structure of the 3D scaffold. While for PLCL membrane, it was challenging to suck $\mathrm{NaBH}_{4}$ solution into the membrane because of its hydrophobic nature, so the PLCL membrane still remained as a thin film. The swelling degree of 2DNFS and 3DNFS was measured by incubating the scaffolds in PBS solution. 3DNFS showed higher swelling degree than that of 2DNFS (Fig. S1, supporting information). 3DNFS possessed loosely-packed multi-layered structure, leading to more voids inside the 3DNFS and a higher swelling degree compared to tightly-packed layers of 2DNFS with small pore between nanofiber. A lower swelling degree may lead to an inadequate supply of nutrients within the 2DNFS, thus limiting cell migration and colonization of scaffolds in vitro and 
A
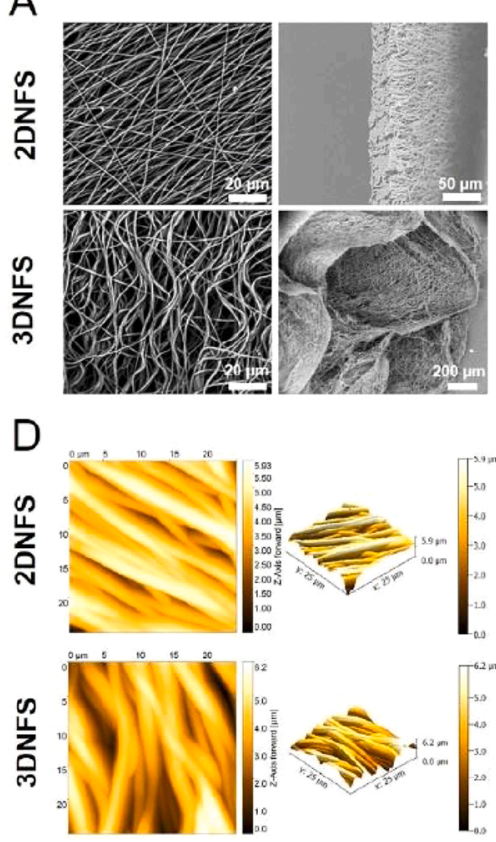

G

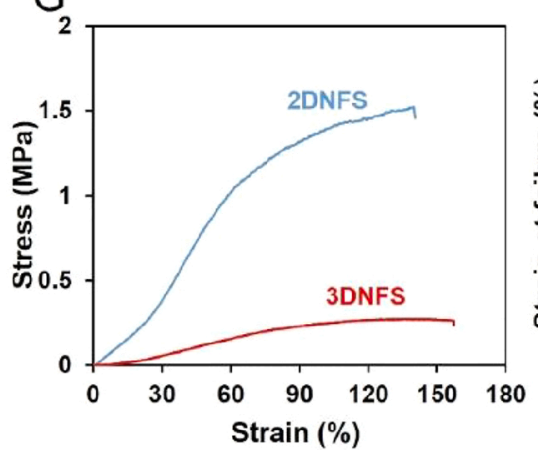

B

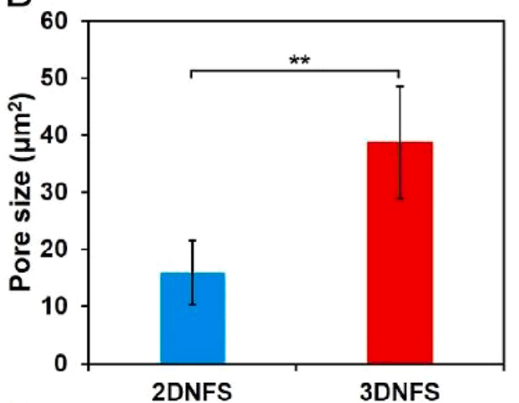

$\mathrm{E}$

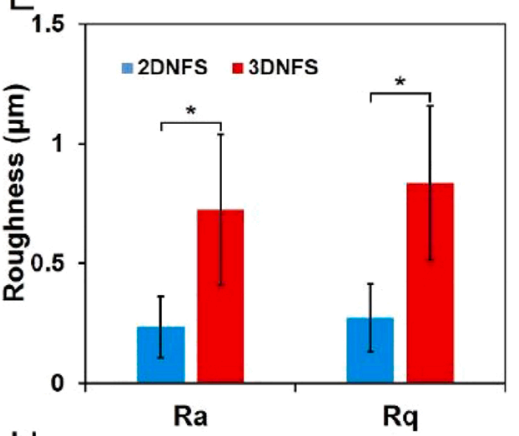

$\mathrm{H}$

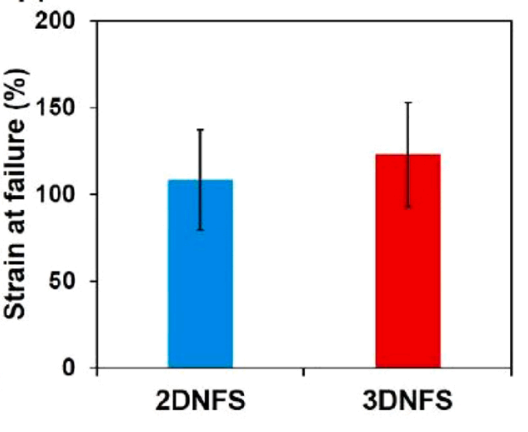

C

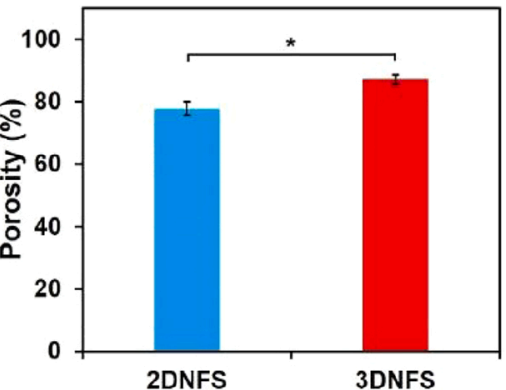

$\mathrm{F}$

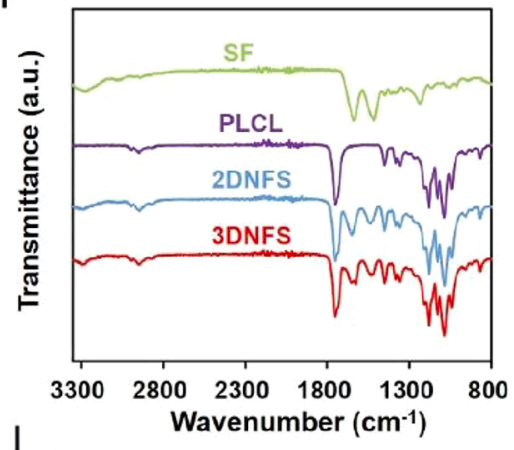

I

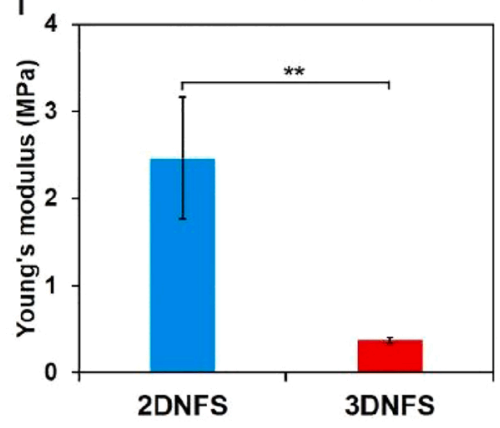

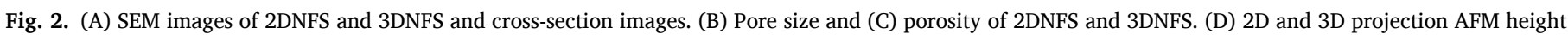

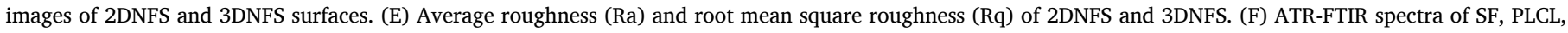

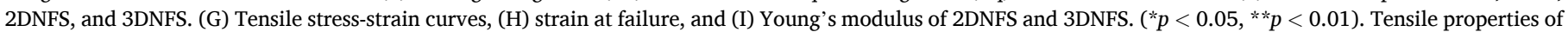
scaffolds were measured in the wet state Samples were soaked in PBS solution for $2 \mathrm{~h}$ (supporting information).

inhibiting tissue ingrowth and vascularization of scaffolds in vivo.

The morphology of 2DNFS and 3DNFS was examined by SEM (Fig. 2A). Both 2DNFS and 3DNFS exhibited aligned nanofiberous morphology. 2DNFS were entirely composed of tightly-packed layers of nanofibers with superficial planar pores. In contrast, 3DNFS maintained the orientation of the fibers and exhibited large pores while fibers twisted to generate a wave-like structure. The cross-sectional images showed that 3DNFS possessed visible loosely-packed multi-layered structure, leading to an increased thickness of 3D scaffold, whereas 2DNFS only exhibited densely-packed cross-section with a small thickness. The results demonstrated that the densely packed 2DNFS were successfully converted into sponge-like 3DNFS with preserved nanotopographic cues, aligned morphology, and reorganized multi-layered structure following gas foaming. Ligament, tendon, cardiac, muscle, and nerve tissues have been shown to possess aligned fibers' morphology [22]. Therefore, these sponge-like 3DNFS maintained aligned morphology which are expected to serve as ECM mimetic materials to promote the regeneration of connective tissues. The pore size in the 3DNFS $\left(38.75 \pm 9.78 \mu \mathrm{m}^{2}\right)$ was significantly larger than that of the 2DNFS (15.98 $\pm 5.64 \mu \mathrm{m}^{2}$ ) (Fig. 2B). Intriguingly, 3DNFS showed significantly high porosity $(87.1 \% \pm 1.5 \%)$ as compared to that $(77.8 \%$ $\pm 2.1 \%$ ) of the 2DNFS (Fig. 2C). Since cell infiltration and tissue regeneration depend on the morphology and structure of nano/microfibers, it is necessary to control the fiber thickness, pore size, and porosity of electrospun scaffolds. The architecture and structure of scaffolds, such as pore size and porosity determine the infiltration of cells. Large pore sizes are recommended for tissue ingrowth, and new tissue formation occurs in the higher porosity areas [23]. Previous studies have shown that the porosity and pore size of electrospun scaffolds can be increased by increasing the thickness of fibers, thus improving the cellular infiltration and inducing macrophage polarization [24]. Therefore, after foaming treatment, 3DNFS afforded a larger pore size and controllable thickness, which is conducive to cell infiltration and tissue regeneration. It has been elucidated that the morphology of scaffolds can be varied by tuning the electrospinning parameters, including the judicious selection of collector (e.g., replacing the traditional 2D flat collector with a 3D collection template afforded controllable porous electrospun PLCL/SF scaffolds) [7]. Another facile approach to afford porous 3D nanofibrous scaffolds is to employ liquid-assisted collection-electrospinning technology. For example, electrospun polyacrylonitrile (PAN) nanofibers were collected directly in a liquid collector containing graphene oxide (GO), and then freeze-dried and thermally treated to obtain fluffy carbon nanofibers/GO aerogels (CNF/GOAs) [25]. Kong and coworkers [26] 
A

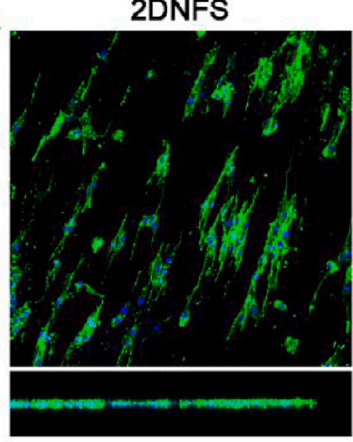

C
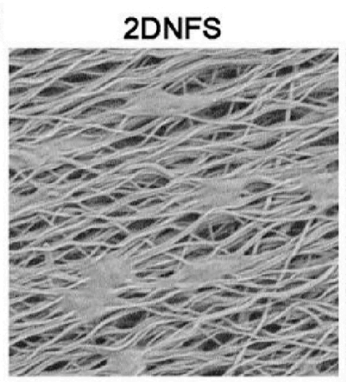

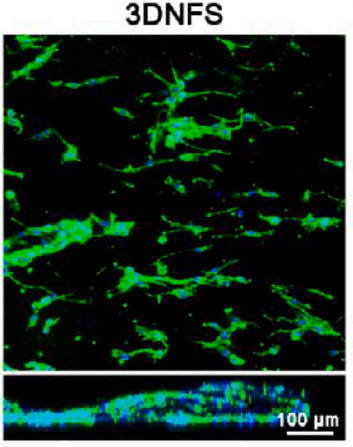

3DNFS

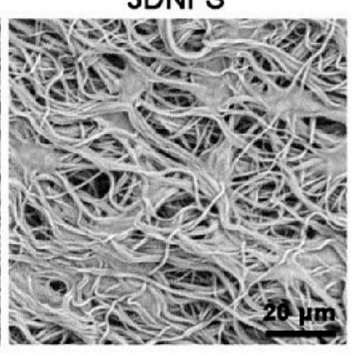

B

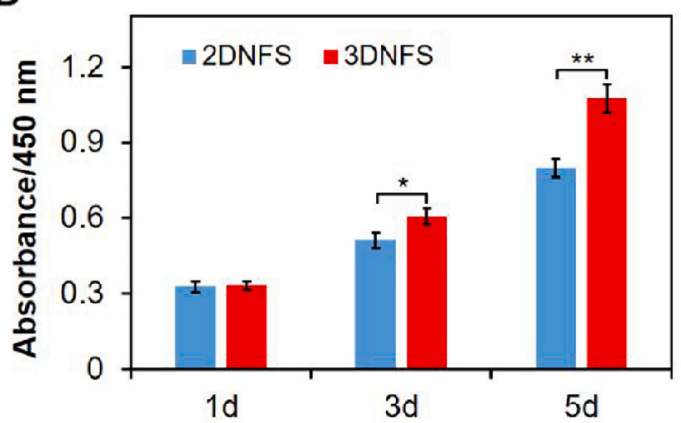

D

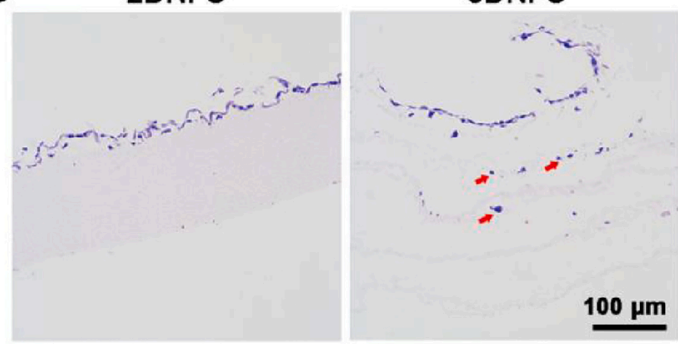

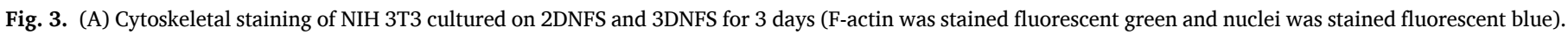

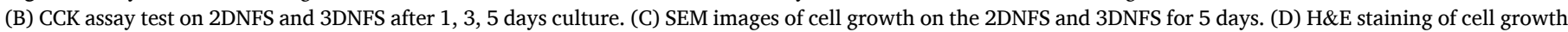
within 2DNFS and 3DNFS for 5 days. (*p<0.05, ** $p<0.01$ ).

fabricated PCL fibers (both thin and thick by electrospinning) and observed that the thicker fibers had large pore diameter than that of the smaller ones. Since ECM fibers are of nano-sizes, the thick fiber size may not fully recapitulate ECM structure. In addition, changing the collector may also be unfavorable to preserve the mechanical stability of the scaffold. On the other hand, gas foaming method can effectively improve the thickness and pore size of scaffolds without substantially affecting the mechanical integrity and nanotopographic cues.

Atomic force microscopy (AFM) was used to evaluate the surface topography and roughness parameters. As shown in Fig. $2 \mathrm{D}$, the $2 \mathrm{D}$ projection and 3D reconstruction height images were able to provide the surface morphology and surface roughness values, where a dark color demonstrated a higher depth on the surface of the scaffold. 3DNFS presented larger peak-to-peak distance and valley-to-valley distance than that of 2DNFS. The quantitative analysis of roughness by AFM is displayed in Fig. 2E, which revealed that the average roughness (Ra) and root mean square roughness (Rq) of 3DNFS significantly increased after expansion. The Ra value of 2DNFS was found to be $0.24 \pm 0.1 \mu \mathrm{m}$, which was increased to $0.72 \pm 0.3 \mu \mathrm{m}$ in 3DNFS. Similarly, an increase in the $\mathrm{Rq}$ value from $0.27 \pm 0.1 \mu \mathrm{m}$ to $0.84 \pm 0.3 \mu \mathrm{m}$ for 2DNFS and 3DNFS, respectively also confirmed that that surface of the 2DNFS became coarser after expansion. These results are consistent with the SEM results, where an increase in the roughness and the twisting of the fibers were caused by the trapped gas bubbles mediated pressure on the surrounding fibers.

The FTIR spectra of SF, PLCL, 2DNFS, and 3DNFS were presented in Fig. $2 \mathrm{~F}$. In the spectrum of the $\mathrm{SF}$, the band at $3295 \mathrm{~cm}^{-1}$ was ascribed to the stretching vibration of $\mathrm{O}-\mathrm{H}$ and $\mathrm{NH}-$. The characteristic bands of amide I and amide II appeared at $1654 \mathrm{~cm}^{-1}$ and $1543 \mathrm{~cm}^{-1}$, respectively. PLCL exhibited characteristic bands at $3069 \mathrm{~cm}^{-1}\left(-\mathrm{CH}_{3}\right.$ stretching vibration) and $2938 \mathrm{~cm}^{-1}\left(-\mathrm{CH}_{2}-\right.$ stretching vibration), while bands appeared at $1756 \mathrm{~cm}^{-1}$ can be attributed to stretching vibration of the carbonyl group ester. The bonds observed at $1454 \mathrm{~cm}^{-1}$ and $1359 \mathrm{~cm}^{-1}$ were ascribed to the $\mathrm{C}-\mathrm{H}$ symmetric and asymmetric vibrations of PLCL. 2DNFS and 3DNFS also displayed all of the aforementioned bands of SF and PLCL, indicating that they have been mixed with each other.

Stress-strain curves of 2DNFS and 3DNFS were displayed in Fig. 2G.
Elongation at break of the 3DNFS slightly increased as compared to 2DNFS, which might be attributed to the highly porous (less dense) and loosely arranged structures of 3DNFS (Fig. 2H). 3DNFS exhibited lower Young's modulus than that of the 2DNFS (Fig. 2I). This was expected since the layered architecture and the presence of voids within the scaffolds may introduce heterogeneity that reduces their ability to support the stress but increase the porosity [27].

\subsection{In vitro cytocompatibility and cell adhesion behavior}

The surface morphology plays a pivotal role in regulating cell growth and directly influences cellular response [28]. To probe the impact of the surface morphology and architecture of scaffolds on cell morphology, we stained the cells growing on different scaffolds by F-actin and nuclei with DAPI and observed the morphology and cytoskeleton under a confocal laser scanning microscope (CLSM). On day 3, most of the cells grew along with the orientation of fibers on 2DNFS and demonstrated a typical fibroblast-like cell morphology compared to a multilateral shape on the 3DNFS. The cross-sectional images showed that 3T3 cells did not only distribute on the surface of the 3DNFS but also proliferated in the multilayer gaps and formed cell layers. In contrast, cells only distributed on the surface of 2DNFS. These results indicated that cells could infiltrate, migrate, and adhere to the adjacent multilayer nanofiber structure of 3DNFS, which were unavailable in 2DNFS. Since the viability of cells is essential to assess the biocompatibility of biomaterials in vitro and in vivo [29], 3T3 cells were seeded on different scaffolds and cultured for 1 , 3 , and 5 days. The cell proliferation was assessed by CCK- 8 assay (Fig. 3B). Cells exhibited high proliferation both on 2DNFS and 3DNFS. However, after culturing from day 3 to day 5, cells seeded on 3DNFS showed significantly high cell proliferation as compared to their $2 \mathrm{D}$ counterparts, revealing that the 3DNFS can promote cell proliferation.

To get further insight into the interaction between cells and substrate in 2DNFS and 3DNFS, the morphology and attachment of the cultured NIH 3T3 cells on scaffolds on day 5 was observed by SEM. As shown in Fig. 3C, it was found that cells spread and displayed aligned spindle shape on the surface of 2DNFS. In contrast, cells were likely to grow into the internal pores of 3DNFS. In the H\&E staining (Fig. 3D), the infiltration of cells in the 3DNFS could be observed, but no cellular 
A

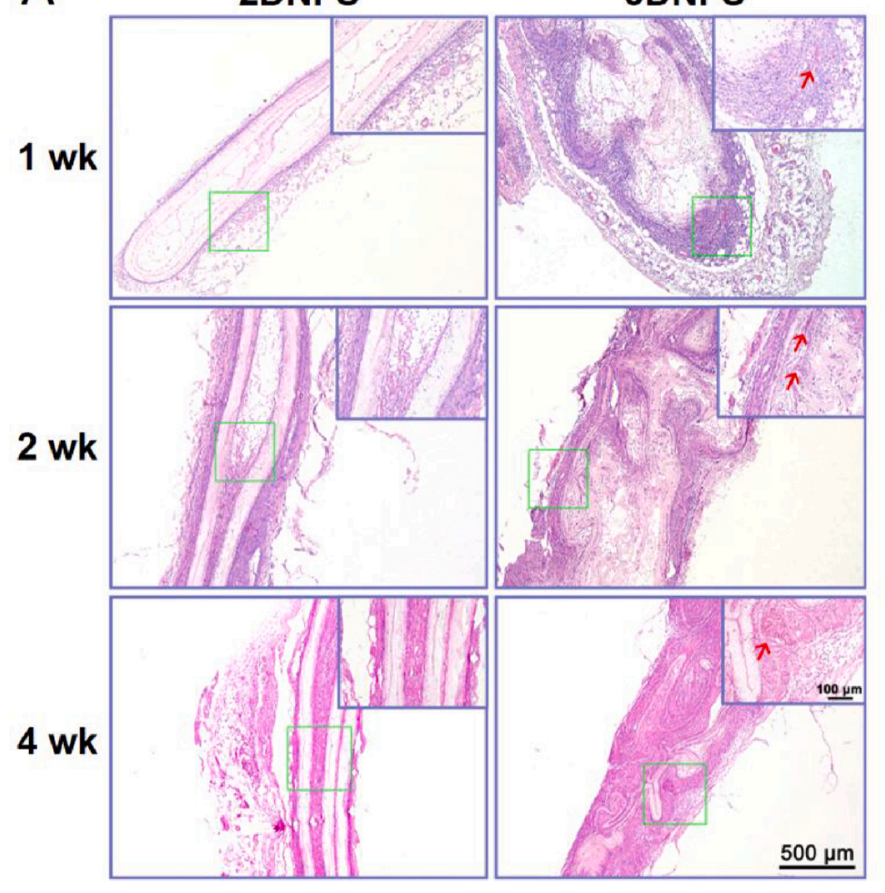

B
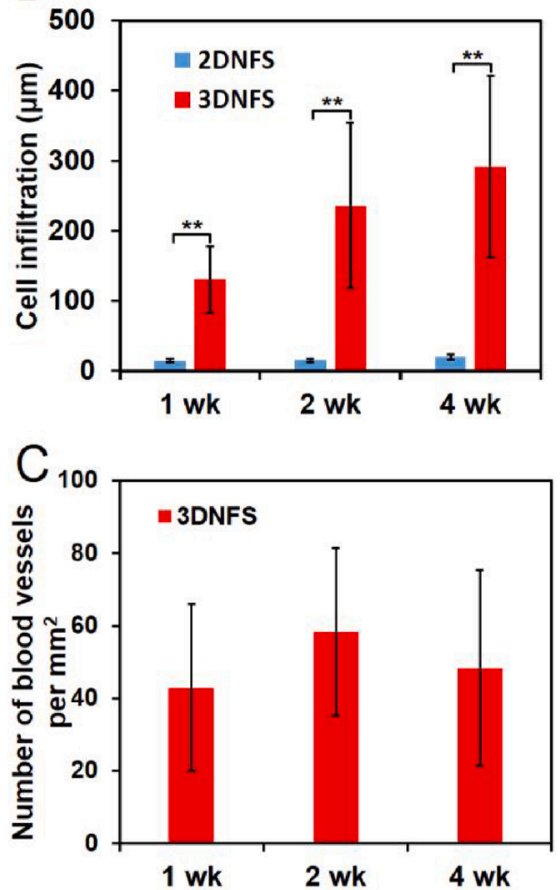

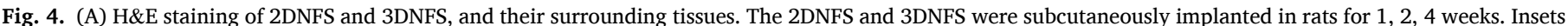

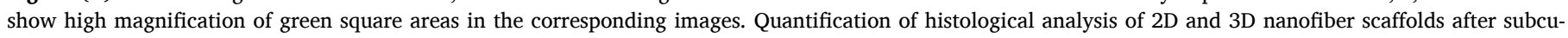
taneous implantation. (B) Cell penetration, (C) number of blood vessels per square millimeter. (*p $<0.05$, ** $p<0.01$ ).
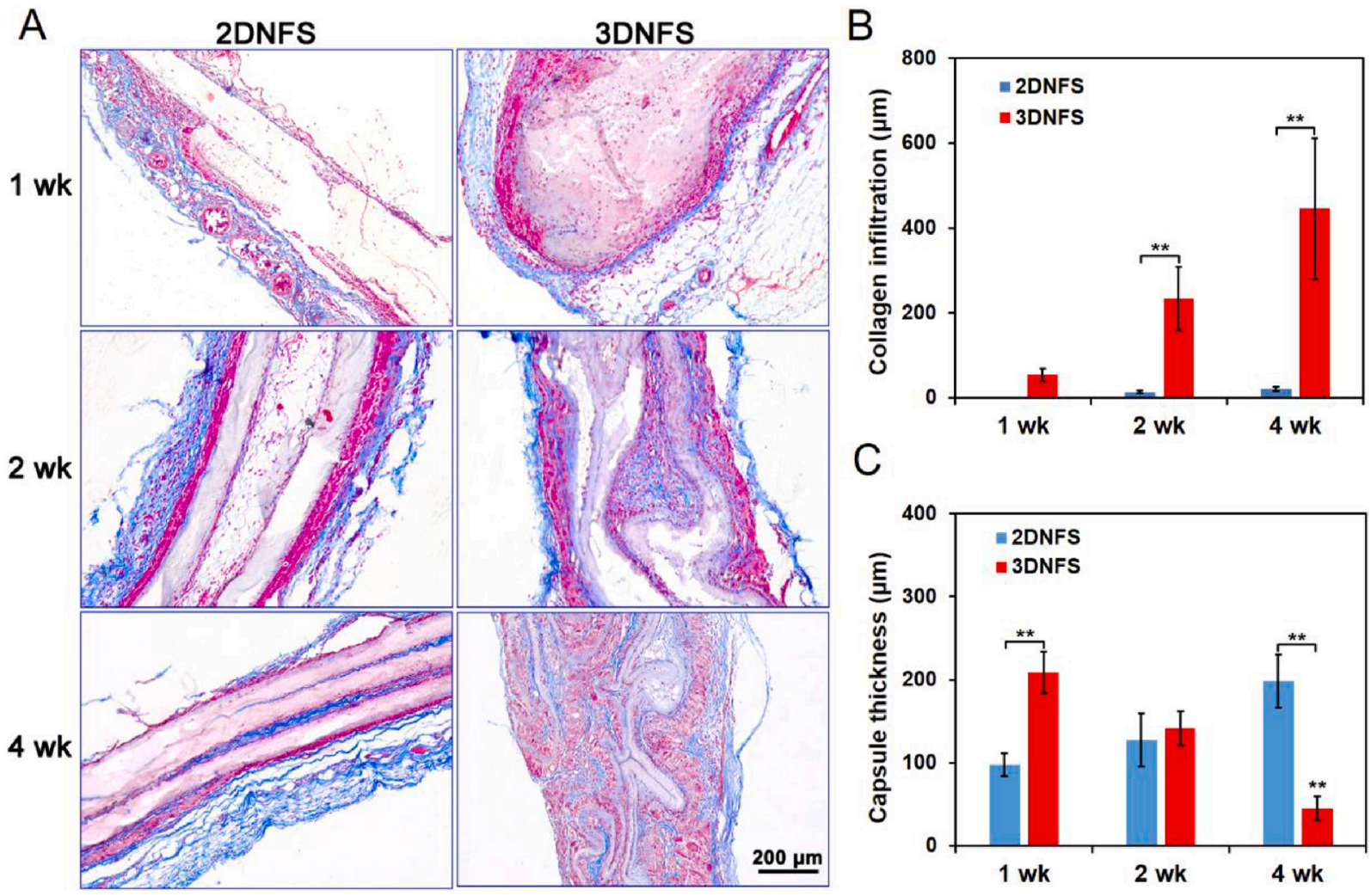

C

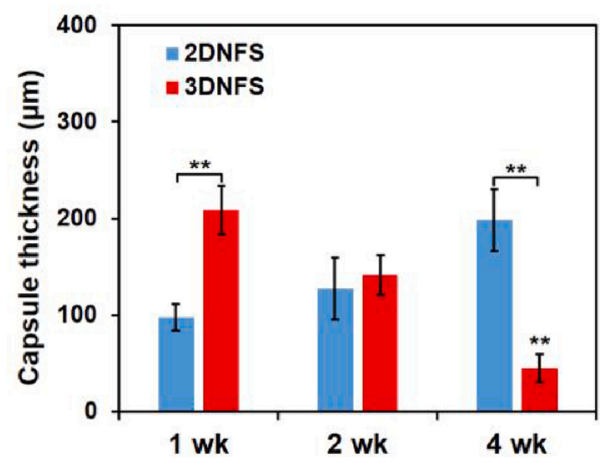

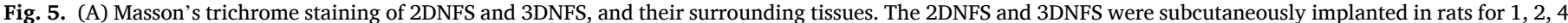

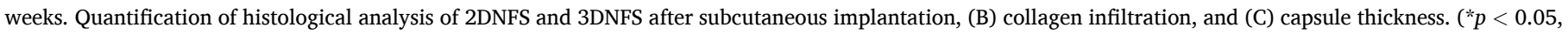
$* * p<0.01$ ). 


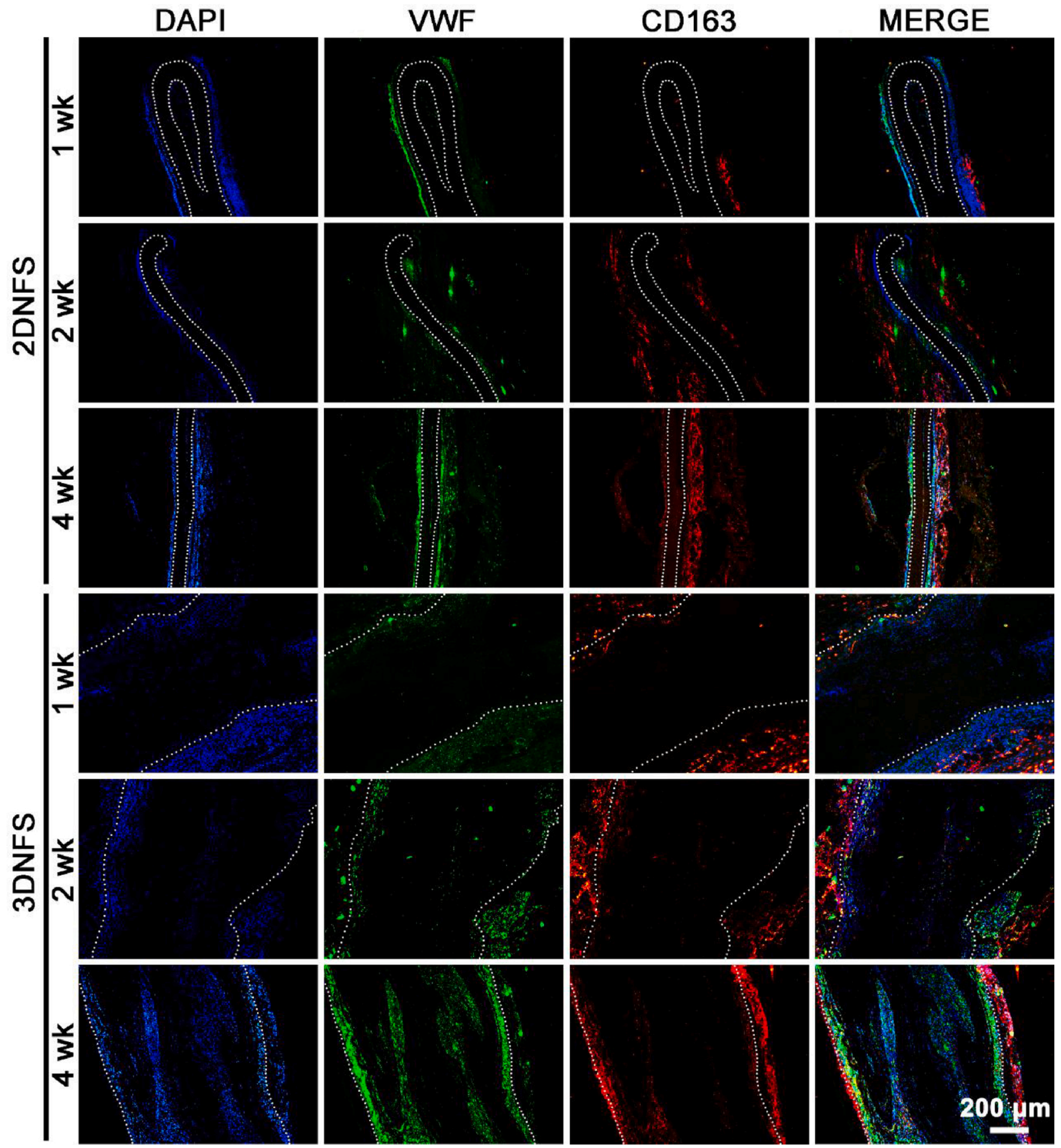

Fig. 6. Immunofluorescent staining for neovascularization (vWF), and macrophage (CD 163) markers of subcutaneous 2DNFS and 3DNFS implants in rats. The white dotted lines delineate the location of the scaffold implant.

infiltration was seen in the unexpanded scaffolds and fibroblasts merely colonized on the apical surface of 2DNFS, further demonstrating that the wavy loosely porous 3DNFS enhanced cell infiltration into the deep layers.

\subsection{Histology analysis}

In order to further investigate the cellular behavior and their infiltration in 2DNFS and 3DNFS in vivo, we implanted scaffolds subcutaneously in rats for up to 1, 2, 4 weeks and performed H\&E and Masson's trichrome (MT) staining. H\&E staining showed that it was difficult for cells to infiltrate into the 2DNFS, but obvious cell infiltration occurred even in the first week in 3DNFS, and cells infiltrated throughout the scaffold in the fourth week (Fig. 4A). The 2DNFS demonstrated a limited increase in the depth of cell infiltration from about $14 \mu \mathrm{m}$ at week 1 , to $15 \mu \mathrm{m}$ at week 2 and $20 \mu \mathrm{m}$ at week 4 (Fig. 4B). Differently, 3DNFS exhibited a steady increase in the depth of the infiltrated cells from 130 $\mu \mathrm{m}$ at week 1, to $236 \mu \mathrm{m}$ at week 2 , and further to $291 \mu \mathrm{m}$ at week 4 . New blood vessel formation within the 3DNFS was observed at week 1 , 2 , and 4, which was not found in 2DNFS. More intriguingly, the thickness of the deposited tissues increased with time on the surface of the 2DNFS. Neovascularization can only be observed on the surface of 2DNFS and a marginal increase of cell infiltration was found inside the 2DNFS. Numbers of blood vessels within the 3DNFS were from 42 vessels $/ \mathrm{mm}^{2}$ at week 1 , to 58 vessels $/ \mathrm{mm}^{2}$ at week 2 , and 48 vessels $/ \mathrm{mm}^{2}$ at week 4 (Fig. 4C). However, no neovascularization was found within the 2DNFS.

Masson's trichrome staining of scaffolds with surrounding tissue is 

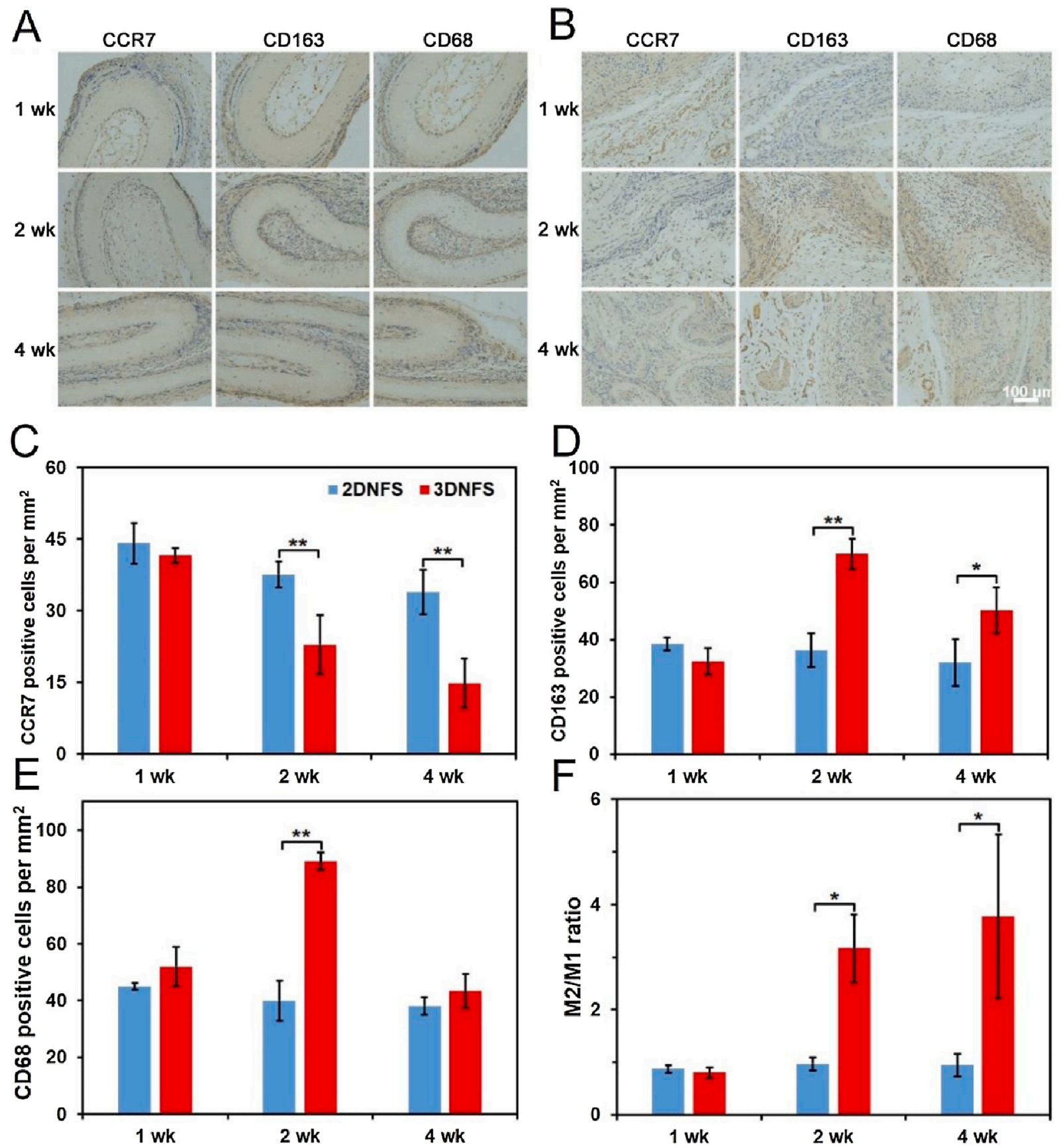

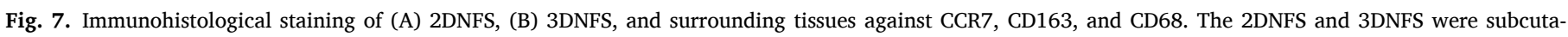

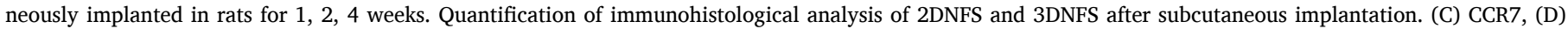

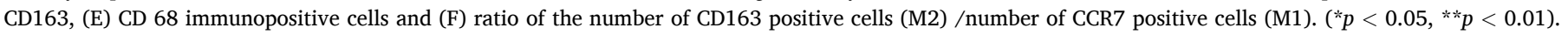

shown in Fig. 5A. The thickness of the collagen capsule on the surface of the 2DNFS increased with time, whereas this trend was not apparent in 3DNFS. More specifically, in 2DNFS, collagen can only be deposited on the surface, and almost no collagen infiltration was exhibited in the fourth week, which was consistent with the result of the H\&E staining. On the other hand, within 3DNFS, we found that the collagen deposition began in the first week, and by the fourth week, the collagen had entered the gaps between nanofiber layers of the scaffold.

Similar to the trend of cell infiltration, the depth of collagen infiltration increased from $53 \mu \mathrm{m}$ at week 1 , to $233 \mu \mathrm{m}$ at week 2 and $445 \mu \mathrm{m}$ at week 4 in 3DNFS (Fig. 5B). In contrast, the depth of collagen infiltration was close to $0 \mu \mathrm{m}$ at week 1 , to $13 \mu \mathrm{m}$ at week 2 , and $20 \mu \mathrm{m}$ at week 4 in 2DNFS. The thickness of collagen fibrous capsules on the surface of 2DNFS increased from $97 \mu \mathrm{m}$ at week 1, to $127 \mu \mathrm{m}$ at week 2 , and further to $198 \mu \mathrm{m}$ at week 4 (Fig. 5C). In contrast, the thickness of the collagen capsule on the surface of 3DNFS was $208 \mu \mathrm{m}, 141 \mu \mathrm{m}$, and $44 \mu \mathrm{m}$ at week 1,2 , and 4 , respectively. Collagen fibrous capsule formation during foreign body reaction (FBR) impedes the normal functioning of an implant. The surface chemistry, physical properties, and morphological aspects of the scaffold impact and regulate the collagen fibrous capsule formation to the implanted scaffold. Porous structure often culminates into a moderate tissue response and permits a rapid healing response. In the present study, 2DNFS were entirely composed of tightly-packed layers of nanofibers with superficial planar pores that prevented cellular and collagen infiltration, resulting into more severe FBR and thicker collagen capsule. On the other hand, 3DNFS possess 
larger porosity as well as aligned nanofiber morphology, resulting into a thinner collagen capsule formation than that of the 2DNFS, indicating a better tissue integration with the host.

Scaffolds and surrounding tissues were sectioned and immunostained for DAPI, VWF, and CD163 (a macrophage marker) to elucidate cell infiltration, neovascularization, and macrophages infiltration. (Fig. 6). Immunostainings also displayed an increase in the cell infiltration, blood vessel formation, and macrophages infiltration within the 3DNFS compared to 2DNFS which were devoid of blood vessels, cell infiltration, and macrophages infiltration owning to the densely packed layers. Neovascularization is an important aspect for in situ tissue regeneration. Pores are essential for vascularization, and higher porosity and multiple layers of scaffolds promote vascularization, which is ascribed to the higher oxygen diffusion and supply of nutrients, thus promoting functional tissue regeneration [30]. Indeed, we did not observe neo-vessel formation in 2DNFS, which is plausibly due to the limited nutrients supply and oxygen diffusion.

To identify the phenotypes of infiltrated macrophages in the scaffolds and the surrounding tissues, we further performed the immunohistological staining of scaffolds with CD68 (a general macrophages marker), CCR7 (a surface marker for M1 macrophages phenotype which encourages inflammation), and CD163 (a surface marker for M2 macrophages phenotype which decreases inflammation and encourages tissue repair). The 2DNFS showed marginal macrophages infiltration (Fig. 7A), while in the 3DNFS, macrophages infiltration was evident at week 1 and increased after that mainly in week 2, which indicated the loosely-packed structure of 3DNFS was conducive to macrophage infiltration (Fig. 7B). We quantified the number of macrophages to identify the phenotypes of infiltrated macrophages. The 2DNFS showed a slight decrease in the number of CCR7 ${ }^{+}$cells from 44 per $\mathrm{mm}^{2}$ at week 1 , to 37 per $\mathrm{mm}^{2}$ at week 2 and 33 per $\mathrm{mm}^{2}$ at week 4 (Fig. 7C). The number of $\mathrm{CD}^{+} 8^{+}$and $\mathrm{CD} 163^{+}$cells, however, was similar from week 1 to week 4 . On the other hand, 3DNFS showed a decreasing number of CCR7 ${ }^{+}$cells from 41 per $\mathrm{mm}^{2}$ at weeks $1-22$ per $\mathrm{mm}^{2}$ at week 2 and 14 per $\mathrm{mm}^{2}$ at week 4 . In the second week, the 3DNFS showed the highest expressions of $\mathrm{CD}^{+} 8^{+}$and $\mathrm{CD}_{163}{ }^{+}$cells, which were 88 per $\mathrm{mm}^{2}$ and 69 per $\mathrm{mm}^{2}$, respectively (Fig. 7D and E). By the fourth week, the number of $\mathrm{CD}^{+} 8^{+}$ and $\mathrm{CD}_{163}{ }^{+}$cells had dropped to 43 per $\mathrm{mm}^{2}$ and 50 per $\mathrm{mm}^{2}$, respectively. The M2/M1 ratios remained constant from week 1 to week 4 for 2DNFS while showed a dramatic increase at week 2 for 3DNFS (Fig. 7F). Our results showed that 3DNFS promoted more M2 macrophage recruitment and polarization within the scaffold than that of 2DNFS, thus facilitating up the repair process, promoting tissue regeneration, and moderating inflammation. Indeed, in multiple disease models, it has been shown that the polarization of the macrophage influences the severity of the disease. 2DNFS showed limited macrophages polarization towards M2 phenotype, which may have led to the longterm repair process. On the other hand, 3DNFS showed more numbers of $\mathrm{CD}_{163^{+}}$cells and fewer numbers of $\mathrm{CCR7}^{+}$cells in scaffolds and surrounding tissues and exhibited a larger ratio of $\mathrm{CD} 163^{+} / \mathrm{CCR}^{+}$cells after 2 weeks than 2DNFS, indicating the resolution of inflammation, polarization of macrophage towards M2 phenotype, and tissue regeneration. Thus, our data demonstrated that the 3DNFS promoted tissue regeneration and modulated FBR.

\section{Conclusions}

We have fabricated three-dimensional hybrid type nanofibrous scaffolds consisting of mechanoelastic and biodegradable PLCL copolymers and SF. This is achieved by expanding 2D electrospun membranes via treatment with a gas foaming agent. PLCL/SF based 3DNFS showed physiochemical stability, nanofibrous morphology, multilayered structure, higher porosity, surface roughness, and preserved nanotopographical architecture than that of 2DNFS following gas foaming as shown by SEM and FTIR spectroscopy. 3DNFS also exhibited good cytocompatibility for tissue formation. This is corroborated by the more cell spreading and growth on the 3DNFS as compared to 2DNFS. The subcutaneous implantation demonstrated thinner collagen capsule formation and more cellular infiltration in expanded scaffolds than that of their unexpanded counterparts. Besides, 3DNFS promotes neovascularization and modulates the inflammatory response, which presumably arises from the larger aperture, aligned morphology, surface roughness, and loosely attached multi-layered structures. Consequently, this strategy allows for the fabrication of 3D-like scaffolds, which may hold great promise to afford 3D tissue models/constructs in vitro, wound dressings, and 3D tissue regeneration.

\section{CRediT authorship contribution statement}

Yujie Chen: Investigation, Methodology, Formal analysis, Writing original draft. Zihao Jia: Investigation, Conceptualization, Data curation. Muhammad Shafiq: Conceptualization, Writing - review \& editing. Xianrui Xie: Investigation, Conceptualization. Xianghao Xiao: Investigation, Conceptualization. Rita Castro: Conceptualization, Data curation. João Rodrigues: Conceptualization, Data curation. Jinglei Wu: Conceptualization, Writing - review \& editing. Guangdong Zhou: Conceptualization, Funding acquisition. Xiumei Mo: Supervision, Funding acquisition, Writing - review \& editing.

\section{Declaration of Competing Interest}

The authors report no declarations of interest.

\section{Acknowledgments}

This research was supported by the National Key Research Program of China (2016YFA0201702 of 2016YFA0201700); The Fundamental Research Funds for the Central Universities (2232019A3-07); National Nature Science Foundation of China (No. 31771023); and Science and Technology Commission of Shanghai Municipality (Nos. 19441902600, 20\$31900900). This work was also supported by FCT-Fundação para a Ciência e a Tecnologia through the CQM Base Fund - UIDB/00674/ 2020, by ARDITI-Agência Regional para o Desenvolvimento da Investigação Tecnologia e Inovação, through the project, M1420-01-0145FEDER-000005 - Centro de Química da Madeira - CQM ${ }^{+}$(Madeira 1420 Program) and M1420-09-5369-FSE-000002 (R. C. Post-Doc Grant). JR also acknowledges the support of Programa de Cooperación Territorial INTERREG V-A MAC 2014-2020, Project Inv2Mac (MAC2/4.6d/ 229).

\section{Appendix A. Supplementary data}

Supplementary material related to this article can be found, in the online version, at doi:https://doi.org/10.1016/j.colsurfb.2021.111637.

\section{References}

[1] J. Wu, Y. Hong, Enhancing cell infiltration of electrospun fibrous scaffolds in tissue regeneration, Bioact. Mater. 1 (2016) 56-64, https://doi.org/10.1016/j. bioactmat.2016.07.001.

[2] C.M. Dumont, D.J. Margul, L.D. Shea, Tissue engineering approaches to modulate the inflammatory milieu following spinal cord injury, Cells Tissues Organs 202 (2016) 52-66, https://doi.org/10.1159/000446646.

[3] B.A. Blakeney, A. Tambralli, J.M. Anderson, A. Andukuri, D.J. Lim, D.R. Dean, H. W. Jun, Cell infiltration and growth in a low density, uncompressed threedimensional electrospun nanofibrous scaffold, Biomaterials 32 (2011) 1583-1590, https://doi.org/10.1016/j.biomaterials.2010.10.056.

[4] S. Soliman, S. Pagliari, A. Rinaldi, G. Forte, R. Fiaccavento, F. Pagliari, O. Franzese, M. Minieri, P. Di Nardo, S. Licoccia, E. Traversa, Multiscale three-dimensional scaffolds for soft tissue engineering via multimodal electrospinning, Acta Biomater. 6 (2010) 1227-1237, https://doi.org/10.1016/j.actbio.2009.10.051.

[5] Y.Z. Cai, G.R. Zhang, L.L. Wang, Y.Z. Jiang, H.W. Ouyang, X.H. Zou, Novel biodegradable three-dimensional macroporous scaffold using aligned electrospun nanofibrous yarns for bone tissue engineering, J. Biomed. Mater. Res. - Part A 100 A (2012) 1187-1194, https://doi.org/10.1002/jbm.a.34063. 
[6] J. Song, G. Zhu, H. Gao, L. Wang, N. Li, X. Shi, Y. Wang, Origami meets electrospinning: a new strategy for 3D nanofiber scaffolds, Bio-Des. Manuf. 1 (2018) 254-264, https://doi.org/10.1007/s42242-018-0027-9.

[7] B. Sun, J. Li, W. Liu, B.M. Aqeel, H. El-Hamshary, S.S. Al-Deyab, X. Mo, Fabrication and characterization of mineralized P(LLA-CL)/SF three-dimensional nanoyarn scaffolds, Iran. Polym. J. 24 (2014) 29-40, https://doi.org/10.1007/s13726-0140297-9.

[8] M.R. Badrossamay, H.A. McIlwee, J.A. Goss, K.K. Parker, Nanofiber assembly by rotary jet-spinning, Nano Lett. 10 (2010) 2257-2261, https://doi.org/10.1021/ nl101355x.

[9] J. Jiang, M.A. Carlson, M.J. Teusink, H. Wang, M.R. MacEwan, J. Xie, Expanding two-dimensional electrospun nanofiber membranes in the third dimension by a modified gas-foaming technique, ACS Biomater. Sci. Eng. 1 (2015) 991-1001, https://doi.org/10.1021/acsbiomaterials.5b00238.

[10] K. Zhang, X. Bai, Z. Yuan, X. Cao, X. Jiao, Y. Li, Y. Qin, Y. Wen, X. Zhang, Layered nanofiber sponge with an improved capacity for promoting blood coagulation and wound healing, Biomaterials 204 (2019) 70-79, https://doi.org/10.1016/j. biomaterials.2019.03.008.

[11] J. Jiang, S. Chen, H. Wang, M.A. Carlson, A.F. Gombart, J. Xie, CO2-expanded nanofiber scaffolds maintain activity of encapsulated bioactive materials and promote cellular infiltration and positive host response, Acta Biomater. 68 (2018) 237-248, https://doi.org/10.1016/j.actbio.2017.12.018.

[12] M.K. Joshi, H.R. Pant, A.P. Tiwari, H.J. kim, C.H. Park, C.S. Kim, Multi-layered macroporous three-dimensional nanofibrous scaffold via a novel gas foaming technique, Chem. Eng. J. 275 (2015) 79-88, https://doi.org/10.1016/j. cej.2015.03.121.

[13] Q. Gao, H. Gu, P. Zhao, C. Zhang, M. Cao, J. Fu, Y. He, Fabrication of electrospun nanofibrous scaffolds with 3D controllable geometric shapes, Mater. Des. 157 (2018) 159-169, https://doi.org/10.1016/j.matdes.2018.07.042.

[14] X. Wang, B. Ding, B. Li, Biomimetic electrospun nanofibrous structures for tissue engineering, Mater. Today 16 (2013) 229-241, https://doi.org/10.1016/j. mattod.2013.06.005.

[15] K. Zhang, X. Bai, Z. Yuan, X. Cao, X. Jiao, Y. Li, Y. Qin, Y. Wen, X. Zhang, Layered nanofiber sponge with an improved capacity for promoting blood coagulation and wound healing, Biomaterials 204 (2019) 70-79, https://doi.org/10.1016/j. biomaterials.2019.03.008.

[16] K. Zhang, X. Mo, C. Huang, C. He, H. Wang, Electrospun scaffolds from silk fibroin and their cellular compatibility, J. Biomed. Mater. Res. - Part A 93 (2010) 976-983, https://doi.org/10.1002/jbm.a.32497.

[17] D. Jin, J. Hu, D. Xia, A. Liu, H. Kuang, J. Du, X. Mo, M. Yin, Evaluation of a simple off-the-shelf bi-layered vascular scaffold based on poly(L-lactide-co$\varepsilon$-caprolactone)/silk fibroin in vitro and in vivo, Int. J. Nanomed. 14 (2019) 4261-4276, https://doi.org/10.2147/IJN.S205569.

[18] B. Sun, Z. Zhou, D. Li, T. Wu, H. Zheng, J. Liu, G. Wang, Y. Yu, X. Mo, Polypyrrolecoated poly(l-lactic acid-co- $\varepsilon$-caprolactone)/silk fibroin nanofibrous nerve guidance conduit induced nerve regeneration in rat, Mater. Sci. Eng. C 94 (2019) 190-199, https://doi.org/10.1016/j.msec.2018.09.021.
[19] X. Xie, Y. Chen, X. Wang, X. Xu, Y. Shen, A.urR. Khan, A. Aldalbahi, A.E. Fetz, G. L. Bowlin, M. El-Newehy, X. Mo, Electrospinning nanofiber scaffolds for soft and hard tissue regeneration, J. Mater. Sci. Technol. 59 (2020) 243-261, https://doi. org/10.1016/j.jmst.2020.04.037.

[20] J. Cai, X. Xie, D. Li, L. Wang, J. Jiang, X. Mo, J. Zhao, A novel knitted scaffold made of microfiber/nanofiber core-sheath yarns for tendon tissue engineering, Biomater. Sci. 8 (2020) 4413-4425, https://doi.org/10.1039/d0bm00816h.

[21] M. Wu, Q. Wang, X. Liu, H. Liu, Biomimetic synthesis and characterization of carbon nanofiber/ hydroxyapatite composite scaffolds, Carbon 51 (2013) 335-345, https://doi.org/10.1016/j.carbon.2012.08.061.

[22] Y. Chen, M. Sha, M. Liu, Y. Morsi, X. Mo, Advanced fabrication for electrospun three-dimensional nanofiber aerogels and scaffolds, Bioact. Mater. 5 (2020) 963-979, https://doi.org/10.1016/j.bioactmat.2020.06.023.

[23] T. Dutta Roy, J.L. Simon, J.L. Ricci, E.D. Rekow, V.P. Thompson, J.R. Parsons, Performance of degradable composite bone repair products made via threedimensional fabrication techniques, J. Biomed. Mater. Res. - Part A 66 (2003) 283-291, https://doi.org/10.1002/jbm.a.10582.

[24] M. Shafiq, Q. Zhang, D. Zhi, K. Wang, D. Kong, D.H. Kim, S.H. Kim, In situ blood vessel regeneration using SP (Substance P) and SDF (Stromal cell-derived factor)$1 \alpha$ peptide eluting vascular grafts, Arterioscler. Thromb. Vasc. Biol. 38 (2018) 117-134, https://doi.org/10.1161/ATVBAHA.118.310934.

[25] Y.Z. Lin, L. Bin Zhong, S. Dou, Z.D. Shao, Q. Liu, Y.M. Zheng, Facile synthesis of electrospun carbon nanofiber/graphene oxide composite aerogels for high efficiency oils absorption, Environ. Int. 128 (2019) 37-45, https://doi.org/ 10.1016/j.envint.2019.04.019.

[26] Z. Wang, Y. Cui, J. Wang, X. Yang, Y. Wu, K. Wang, X. Gao, D. Li, Y. Li, X.L. Zheng, Y. Zhu, D. Kong, Q. Zhao, The effect of thick fibers and large pores of electrospun poly( $\varepsilon$-caprolactone) vascular grafts on macrophage polarization and arterial regeneration, Biomaterials 35 (2014) 5700-5710, https://doi.org/10.1016/j. biomaterials.2014.03.078.

[27] F.A. Soares, S.M.B. Nachtigall, Effect of chemical and physical foaming additives on the properties of PP/wood flour composites, Polym. Test. 32 (2013) 640-646, https://doi.org/10.1016/j.polymertesting.2013.02.009.

[28] D.D. Deligianni, N.D. Katsala, P.G. Koutsoukos, Y.F. Missirlis, Effect of surface roughness of hydroxyapatite on human bone marrow cell adhesion, proliferation, differentiation and detachment strength, Biomaterials 22 (2000) 87-96, https:// doi.org/10.1016/S0142-9612(00)00174-5.

[29] H.R. Pant, H.J. Kim, L.R. Bhatt, M.K. Joshi, E.K. Kim, J.I. Kim, A. Abdal-Hay, K. S. Hui, C.S. Kim, Chitin butyrate coated electrospun nylon-6 fibers for biomedical applications, Appl. Surf. Sci. 285 (2013) 538-544, https://doi.org/10.1016/j. apsusc.2013.08.089.

[30] B.M. Whited, J.R. Whitney, M.C. Hofmann, Y. Xu, M.N. Rylander, Pre-osteoblast infiltration and differentiation in highly porous apatite-coated PLLA electrospun scaffolds, Biomaterials 32 (2011) 2294-2304, https://doi.org/10.1016/j. biomaterials.2010.12.003. 